Typeset with jpsj2.cls $<$ ver.1.2 $>$

LETTER

\title{
Formation of non-unitary state near the upper-critical field of $\mathrm{Sr}_{2} \mathrm{RuO}_{4}$
}

\author{
Masafumi Udagawa, Youichi Yanase, and Masao Ogata \\ Department of Physics, University of Tokyo, Hongo, Tokyo 113-0033, Japan
}

(Received November 21, 2018)

\begin{abstract}
We have studied the superconducting state of $\mathrm{Sr}_{2} \mathrm{RuO}_{4}$ under a magnetic field parallel to the superconducting plane. We show that due to a weak spin-orbit coupling, non-unitary $\mathrm{k}_{y}(\hat{\mathbf{z}}-i \alpha \hat{\mathbf{y}})$ state is formed right at $\mathrm{H}_{c 2}$, then changes to unitary $\mathrm{k}_{y} \hat{\mathbf{z}}$ state, as a magnetic field is lowered. In terms of this crossover, we address the origin of the observed double peaks of specific heat and the disappearance of the double peaks at low fields.

KEYWORDS: $\mathrm{Sr}_{2} \mathrm{RuO}_{4}$, two-component order parameter, specific heat, tricritical point, nonunitary state
\end{abstract}

$\mathrm{Sr}_{2} \mathrm{RuO}_{4}$ has been intensively studied in the last years, and its superconducting properties are well understood as to a zero magnetic field state. ${ }^{1,2} \mu \mathrm{SR}$ experiment ${ }^{3}$ and a microscopic calculation $^{4,5}$ consistently support the chiral triplet superconductivity with the order parameter, denoted as $\mathbf{d}(\mathbf{k}) \propto\left(k_{x} \pm i k_{y}\right) \hat{\mathbf{z}}$. Recently, momentum dependence of the gap amplitude was also determined with a high-precision measurement of the angle-resolved specific heat. ${ }^{6}$

However, the two-component order parameter $\mathbf{d}(\mathbf{k}) \propto\left(k_{x} \pm i k_{y}\right) \hat{\mathbf{z}}$ seems to contradict a behavior under the in-plane magnetic field. As Agterberg pointed out, the in-plane magnetic field lifts the degeneracy between $k_{x} \hat{\mathbf{z}}$ and $k_{y} \hat{\mathbf{z}} .{ }^{7}$ Given a magnetic field is applied parallel to the $x$-axis, only one component with a shorter coherence length, say $k_{y} \hat{\mathbf{z}}$, is condensed near the upper-critical field $\left(H_{c 2}\right)$. Since $k_{y} \hat{\mathbf{z}}$ is symmetric with respect to a reflection about the $y$-axis while $\left(k_{x} \pm i k_{y}\right) \hat{\mathbf{z}}$ are not, a phase transition line must exist between $H=0$ and $H=H_{c 2}$ for all temperatures below $T_{c}$.

Actually, the observed double peaks of the specific heat, ${ }^{8,9}$ the thermal conductivity, ${ }^{10}$ and the ac-susceptibility ${ }^{11}$ suggest an existence of a second-order phase transition. However, these observations show several inconsistencies with the prediction by Agterberg. ${ }^{7}$ Firstly, the second peak was observed at much higher field than the theoretical estimate. Secondly, the second peak line terminates at $(H, T) \sim(1.2 \mathrm{~T}, 0.8 \mathrm{~K})$ and merges into the $H_{c 2}$ line. In particular, the termination of the second peak line suggests that the zero-field $\left(k_{x} \pm i k_{y}\right) \hat{\mathbf{z}}$ state can be smoothly continued up to $\mathrm{H}_{c 2}$ near $\mathrm{T}_{c}$, thus inconsistent with the symmetry argument above.

Since the position of transition line is susceptible to the form of the pairing function, etc., it may be possible to attribute the observed peaks to Agterberg's phase transition. ${ }^{12}$ However, in this paper, we would like to address this problem from a different standpoint, 
i.e., by considering the $\mathbf{d}$-vector degrees of freedom. In $\mathrm{Sr}_{2} \mathrm{RuO}_{4}$, at zero field, $\mathbf{d}$-vector is fixed to be parallel to $\hat{\mathbf{z}}$ due to the spin-orbit interaction between $3 d$ electrons forming the three Fermi surfaces. ${ }^{5}$ However, the energy splittings between the stable $(\mathrm{A})\left(k_{x} \pm i k_{y}\right) \hat{\mathbf{z}}$ and unstable $(\mathrm{B}) k_{x} \hat{\mathbf{x}} \pm k_{y} \hat{\mathbf{y}}$ or $(\mathrm{C}) k_{x} \hat{\mathbf{y}} \pm k_{y} \hat{\mathbf{x}}$ configurations are considerably small. According to the microscopic calculation, it was estimated to be less than $0.01 T_{c}{ }^{5}$ Such a small energy difference naturally results from the fact that spin-orbit interaction affects the superconductivity only in the order of $\left(\frac{\lambda}{W}\right)^{2}$, where $\lambda$ is the LS coupling constant, and $W$ is the band-width. These tiny energy differences are also supported by the NMR experiment with the magnetic field parallel to $\hat{\mathbf{z}}^{13}$ There the $\mathbf{d}$-vector is shown to flip at $H \sim 200$ Oe, which means the states (A)-(C) are degenerate within $\sim 0.001 T_{c}$.

First, let us explain qualitatively the physical mechanism of the double peaks which is proposed in this paper. The quasi-degeneracy of order parameters enables the $k_{y} \hat{\mathbf{y}}$ component to mix with the most stable $k_{y} \hat{\mathbf{z}}$. Actually, under a magnetic field, the mixing is caused by the spin-dependent chemical potential shift due to Zeeman coupling with the external field. If the density of states(DOS) becomes larger with increasing energy as in $\mathrm{Sr}_{2} \mathrm{RuO}_{4}$, the Cooper pairs with spins parallel to $\mathbf{H}$ are easier to form than those with anti-parallel spins. This effect is especially strong in $\mathrm{Sr}_{2} \mathrm{RuO}_{4}$ due to the Van Hove singularity just above the Fermi energy. Therefore, the spin-polarized Cooper pairs, i.e., $k_{y}(\hat{\mathbf{z}}-i \alpha \hat{\mathbf{y}})$ state becomes stable near $H_{c 2}$, at the expense of part of spin-orbit coupling energy. As the magnetic field is lowered, however, the $k_{y}(\hat{\mathbf{z}}-i \alpha \hat{\mathbf{y}})$ state becomes unstable, since a non-unitary state generally loses condensation energy. As a result, a crossover to the unitary $k_{y} \hat{\mathbf{z}}$ state occurs accompanying a large entropy release. This crossover naturally leads to the double peaks of specific heat as observed.

We also explain the disappearance of double peaks near $T=T_{c}$ at low magnetic fields in the same mechanism. The crossover is observable only when the energy gain due to spin-polarization of Cooper pairs surpasses the spin-orbit coupling energy. While the spinpolarization becomes stronger with increasing magnetic field, the spin-orbit coupling energy does not change with field. Therefore, the double peaks are observed only at low temperatures when $H_{c 2}$ is high enough.

In the following, we demonstrate our ideas given above by using the following effective Hamiltonian,

$$
\begin{gathered}
H=\sum_{\mathbf{k}, s}\left(\xi_{\mathbf{k}}-\frac{1}{2} g \mu_{B} \mathbf{s} \cdot \mathbf{H}\right) C_{\mathbf{k}, s}^{\dagger} C_{\mathbf{k}, s} \\
-\frac{1}{2} g_{1} \sum_{\mathbf{k}, \mathbf{k}^{\prime}, s}\left[\left(\Phi_{x}(\mathbf{k}) \Phi_{x}\left(\mathbf{k}^{\prime}\right)+\Phi_{y}(\mathbf{k}) \Phi_{y}\left(\mathbf{k}^{\prime}\right)\right) C_{\mathbf{k}, s}^{\dagger} C_{-\mathbf{k}, s}^{\dagger} C_{-\mathbf{k}^{\prime}, s} C_{\mathbf{k}^{\prime}, s}\right] \\
-\frac{1}{2} g_{2} \sum_{\mathbf{k}, \mathbf{k}^{\prime}, s}\left[i s_{z}\left(\Phi_{x}(\mathbf{k}) \Phi_{y}\left(\mathbf{k}^{\prime}\right)-\Phi_{y}(\mathbf{k}) \Phi_{x}\left(\mathbf{k}^{\prime}\right)\right) C_{\mathbf{k}, s}^{\dagger} C_{-\mathbf{k}, s}^{\dagger} C_{-\mathbf{k}^{\prime}, s} C_{\mathbf{k}^{\prime}, s}\right]
\end{gathered}
$$




$$
\begin{array}{r}
-g_{3} \sum_{\mathbf{k}, \mathbf{k}^{\prime}}\left[\left(\Phi_{x}(\mathbf{k}) \Phi_{x}\left(\mathbf{k}^{\prime}\right)+\Phi_{y}(\mathbf{k}) \Phi_{y}\left(\mathbf{k}^{\prime}\right)\right) C_{\mathbf{k}, \uparrow}^{\dagger} C_{-\mathbf{k}, \downarrow}^{\dagger} C_{-\mathbf{k}^{\prime}, \downarrow} C_{\mathbf{k}^{\prime}, \uparrow}\right] \\
-\frac{1}{2} g_{4} \sum_{\mathbf{k}, \mathbf{k}^{\prime}}\left[\left(\Phi_{x}(\mathbf{k}) \Phi_{x}\left(\mathbf{k}^{\prime}\right)-\Phi_{y}(\mathbf{k}) \Phi_{y}\left(\mathbf{k}^{\prime}\right)\right) C_{\mathbf{k}, \uparrow}^{\dagger} C_{-\mathbf{k}, \uparrow}^{\dagger} C_{-\mathbf{k}^{\prime}, \downarrow} C_{\mathbf{k}^{\prime}, \downarrow}+\text { h.c. }\right] .
\end{array}
$$

Here $C_{\mathbf{k}, s}$ represents electron annihilation operator for $\gamma$-band. $\mathbf{s}$ is the Pauli spin matrix and $\uparrow(\downarrow)$ means the spin direction (anti)parallel to $\hat{\mathbf{z}}$. The pairing functions $\Phi_{x}(\mathbf{k})$ and $\Phi_{y}(\mathbf{k})$, and the coupling constants $g_{1}-g_{4}$ are obtained from the results in the third-order perturbation calculation on a Hubbard-like model. ${ }^{5} \Phi_{x}(\mathbf{k})$ and $\Phi_{y}(\mathbf{k})$ have the same rotational properties as $\mathrm{k}_{x}$ and $\mathrm{k}_{y}$ under the $D_{4 h}$ point group of $\mathrm{Sr}_{2} \mathrm{RuO}_{4}$. The detailed forms of these function do not matter in the subsequent analysis. The kinetic energy term $\xi_{\mathbf{k}}$ is expressed with the two-dimensional tight-binding model for the $\gamma$-band, $\xi_{\mathbf{k}}=-2 t_{z}\left(\cos \left(k_{x}\right)+\cos \left(k_{y}\right)\right)-$ $4 t_{z}^{\prime} \cos \left(k_{x}\right) \cos \left(k_{y}\right)-\mu_{z}$, with $\frac{t_{z}^{\prime}}{t_{z}}=0.4$ and $\mu_{z} \sim 1.555$. Note that we are interested in a sufficiently high magnetic field where contributions from the $\alpha$ - and $\beta$-bands are negligible.

Values of $g_{j}$ are determined in order to reproduce the transition temperature, $T_{c}$, of each order parameter. $T_{c}$ of each order parameter component is given by the combination of $g_{j}$ as shown in Table. I. When the spin-orbit coupling is absent, i.e., $\lambda=0$, all the states (A)-(C) are degenerate, i.e., $g_{1}=g_{3}$ and $g_{4}=0$. However, the spin-orbit coupling lifts this degeneracy and $(\mathrm{A})\left(\Phi_{x}(\mathbf{k}) \pm i \Phi_{y}(\mathbf{k})\right) \hat{\mathbf{z}}$ shows the highest $T_{c}$, then $g_{3}$ becomes dominant. $g_{3}-g_{1}>0$ and $g_{4}$ are originated from the spin-orbit interaction and much smaller than $g_{3} . g_{4}$ determines the relative stability of $(\mathrm{B}) \Phi_{x}(\mathbf{k}) \hat{\mathbf{x}} \pm \Phi_{y}(\mathbf{k}) \hat{\mathbf{y}}$ and $(\mathrm{C}) \Phi_{x}(\mathbf{k}) \hat{\mathbf{y}} \pm \Phi_{y}(\mathbf{k}) \hat{\mathbf{x}}$. Although the two-fold degeneracies in (B) and in (C) are lifted by a weak interaction through the $g_{2}$ channel, $g_{2}$ will play no role in the subsequent analysis and we put $g_{2}=0$ for simplicity. Below, we use $\frac{1}{N(0) g_{1}}-\frac{1}{N(0) g_{3}}=0.001$ and $\frac{1}{N(0)\left(g_{1}+g_{4}\right)}-\frac{1}{N(0)\left(g_{1}-g_{4}\right)}=0.0015$ as typical parameters.

\begin{tabular}{|c|c|c|}
\hline & pairing function & $\mathrm{G}$ \\
\hline$(\mathrm{A})$ & $\left(\Phi_{x}(\mathbf{k}) \pm i \Phi_{y}(\mathbf{k}) \hat{\mathbf{z}}\right.$ & $\mathrm{g}_{3}$ \\
\hline$(\mathrm{B})$ & $\Phi_{x}(\mathbf{k}) \hat{\mathbf{x}} \pm \Phi_{y}(\mathbf{k}) \hat{\mathbf{y}}$ & $\mathrm{g}_{1}-\mathrm{g}_{4}$ \\
\hline$(\mathrm{C})$ & $\Phi_{x}(\mathbf{k}) \hat{\mathbf{y}} \pm \Phi_{y}(\mathbf{k}) \hat{\mathbf{x}}$ & $\mathrm{g}_{1}+\mathrm{g}_{4}$ \\
\hline
\end{tabular}

Table I. Classification of pairing functions and the corresponding coupling constants. The transition temperature of each state is proportional to $\exp \left[-\frac{1}{N(0) G}\right]$ with $\mathrm{N}(\epsilon)$ being the density of states at $\epsilon=\epsilon_{F}$.

In order to study the superconducting state close to $H_{c 2}$, we derive Ginzburg-Landau free energy from eq. (1) in the weak-coupling approximation, as is legitimate for $\mathrm{Sr}_{2} \mathrm{RuO}_{4}$ $\left(T_{c} / \epsilon_{F} \sim 10^{-4}\right)$. We ignore the $\mathbf{d}$-vector component parallel to $\mathbf{H}=\left(H \cos \phi_{0}, H \sin \phi_{0}, 0\right)$, which is suppressed due to the Pauli-paramagnetic effect. Furthermore, we only consider the components with short coherence lengths, i.e., those with the pairing function $\Phi_{\|}(\mathbf{k}) \equiv$ 


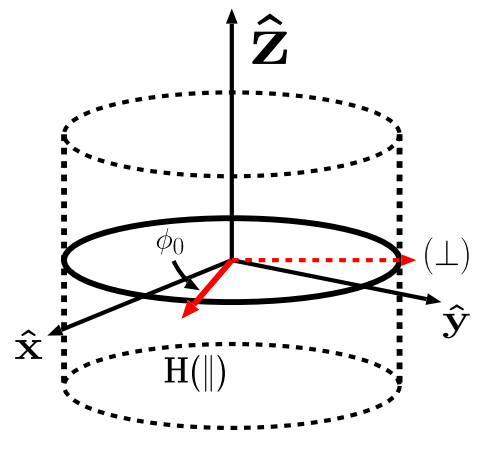

Fig. 1. The direction of magnetic field $\mathbf{H}$. The direction parallel to $\mathbf{H}$ is termed as $\|$. The direction perpendicular to both $\mathbf{H}$ and $\hat{\mathbf{z}}$ is termed as $\perp$.

$\cos \phi_{0} \Phi_{y}(\mathbf{k})+\sin \phi_{0} \Phi_{x}(\mathbf{k}) .{ }^{14}$ By taking the spin axis parallel to $\mathbf{H}$, we obtain

$$
\begin{gathered}
\frac{F_{G L}}{N(0)}=t\left(\left|d_{\perp}\right|^{2}+\left|d_{z}\right|^{2}\right)+\alpha_{s o}\left|d_{\perp}\right|^{2}+C_{2 \perp}\left[\left|\partial_{\perp} d_{\perp}\right|^{2}+\left|\partial_{\perp} d_{z}\right|^{2}\right]+C_{2 z}\left[\left|\partial_{z} d_{\perp}\right|^{2}+\left|\partial_{z} d_{z}\right|^{2}\right] \\
-i \alpha_{s p}\left(d_{\perp} d_{z}^{*}-d_{z} d_{\perp}^{*}\right)+\frac{C_{4}}{2}\left[\left(\left|d_{\perp}\right|^{2}+\left|d_{z}\right|^{2}\right)^{2}+\left|\left(d_{\perp} d_{z}^{*}-d_{z} d_{\perp}^{*}\right)\right|^{2}\right] \\
\alpha_{s o}=\frac{1}{N(0)}\left(\frac{1}{g_{1}+g_{4} \cos ^{2}\left(2 \phi_{0}\right)}-\frac{1}{g_{3}}\right) \\
\alpha_{s p}=\frac{1}{4 N(0)} \int d \xi_{\mathbf{k}}\left[N\left(\xi_{\mathbf{k}}+\frac{1}{2} g \mu_{B} H\right)-N\left(\xi_{\mathbf{k}}-\frac{1}{2} g \mu_{B} H\right)\right] \frac{\tanh \left(\frac{\xi_{\mathbf{k}}}{2 T}\right)}{\xi_{\mathbf{k}}}
\end{gathered}
$$

where the order parameter $d_{\perp}$ and $d_{z}$ are defined as

$$
\begin{aligned}
& d_{\perp}=\frac{1}{2 i}\left(\Delta_{\uparrow \uparrow}+\Delta_{\downarrow \downarrow}\right), \\
& d_{z}=\frac{1}{2}\left(\Delta_{\uparrow \uparrow}-\Delta_{\downarrow \downarrow}\right) .
\end{aligned}
$$

Here, $\Delta_{\uparrow \uparrow(\downarrow \downarrow)}$ means the order parameter component for the electron pairs with (anti)parallel spins to the magnetic field $\mathbf{H}$. The gauge invariant differential operator is given by $\partial_{j}=$ $\nabla_{j}-i \frac{2 e}{c} A_{j}(j=\perp, z)$, where the subscript $\perp$ means the direction perpendicular to both $\mathbf{H}$ and $\hat{\mathbf{z}}$ (see Fig. 1). The coefficients in eq. (2) are $t=\frac{T-T_{c 0}}{T_{c 0}}$ with $T_{c 0}$ being the transition temperature at $\mathbf{H}=0$ for $(\mathrm{A})$, and $C_{2 z(\perp)}=\frac{7 \zeta(3)}{32 \pi^{2} T_{c 0}^{2}}\left\langle v_{F z(\perp)}^{2} \Phi_{\|}(\mathbf{k})^{2}\right\rangle_{F S}, C_{4}=\frac{7 \zeta(3)}{16 \pi^{2} T_{c 0}^{2}}\left\langle\Phi_{\|}(\mathbf{k})^{4}\right\rangle_{F S}$ with $\langle\cdots\rangle_{F S}$ being the average over the Fermi surface.

The two parameters $\alpha_{s o}$ and $\alpha_{s p}$ play important roles in the subsequent analysis. $\alpha_{s o}$ is originated from the spin-orbit interaction, which stabilizes $d_{z}$ compared with $d_{\perp}$. On the other hand, $\alpha_{s p}$ stems from the spin-polarizing effect, which favors $\Delta_{\uparrow \uparrow}$ compared with $\Delta_{\downarrow \downarrow}$. To understand this, it is helpful to rewrite this term as $-i \alpha_{s p}\left(d_{\perp} d_{z}^{*}-d_{z} d_{\perp}^{*}\right)=-\frac{1}{2} \alpha_{s p}\left(\left|\Delta_{\uparrow \uparrow}\right|^{2}-\mid\right.$ $\left.\Delta_{\downarrow \downarrow}\right|^{2}$ ). As explained in the Introduction, this term comes from the spin-dependent chemical potential shift due to the gradient of density of states. For the spatial dependence of the order parameters, we introduce the parameters $r, \theta$, and $\chi$, and put $\mathrm{d}_{z}=r \sin \theta \Psi(\mathbf{r})$ and 
$\mathrm{d}_{\perp}=r \cos \theta e^{-i \chi} \Psi(\mathbf{r})\left(0 \leq \theta \leq \frac{\pi}{2}\right)$, where $\Psi(\mathbf{r})$ is the wave function in the lowest Landau level, satisfying the relation $\left(C_{2 \perp} \partial_{\perp}^{2}+C_{2 z} \partial_{z}^{2}\right) \Psi(\mathbf{r})=\sqrt{C_{2 \perp} C_{2 z}} \frac{2 e}{c} H \Psi(\mathbf{r}) \equiv \frac{H}{H_{0}} \Psi(\mathbf{r})$ and being normalized as $\int \frac{d \mathbf{r}}{V}|\Psi(\mathbf{r})|^{2}=1$. Here, $H_{0}$ is the upper-critical field at $T=0$ when $\alpha_{s p}=$ 0 . Considering that $H_{0} \sim 1.5$ Tesla for $\mathrm{Sr}_{2} \mathrm{RuO}_{4}$, we set $\frac{g \mu_{B} H_{0}}{2 T_{c 0}}=0.5$. Using these order parameters, we obtain the spatial average of $F_{G L}\left(\bar{F}_{G L}(r, \theta, \chi)\right)$ and minimize $\bar{F}_{G L}$ with respect to $r$ and $\chi$. The results are

$$
\begin{gathered}
\bar{F}_{G L}\left(r_{\text {min }}, \theta, \chi_{\text {min }}\right) \propto-\frac{\left(t_{c}-t-\sqrt{\left(2 \alpha_{s p}\right)^{2}+\alpha_{s o}^{2}} \sin ^{2}\left(\theta-\theta_{c}\right)\right)^{2}}{1+\sin ^{2}(2 \theta)}, \\
r_{\text {min }}^{2}=\frac{|t|-\alpha_{s o} \cos ^{2} \theta+\alpha_{s p} \sin (2 \theta)-\frac{H}{H_{0}}}{C_{4}|\bar{\Psi}|^{4}\left(1+\sin ^{2}(2 \theta)\right)} \\
\chi_{\text {min }}=\frac{\pi}{2},
\end{gathered}
$$

where

$$
\begin{gathered}
\theta_{c}=\frac{\pi}{4}+\frac{1}{2} \tan ^{-1}\left(\frac{\alpha_{s o}}{2 \alpha_{s p}}\right), \\
t_{c}=-\frac{H}{H_{0}}+\sqrt{\alpha_{s p}^{2}+\left(\frac{\alpha_{s o}}{2}\right)^{2}}-\frac{\alpha_{s o}}{2} .
\end{gathered}
$$

The critical temperature $t=t_{c}$ was found by putting $r^{2}=0$ and $\theta=\theta_{c}$ in eq. (8). Here, $\theta_{c}$ expresses the spin-polarization of Cooper pairs at $t=t_{c}$, and its value is determined from the competition between $\alpha_{s o}$ and $\alpha_{s p}$ as in eq. (10). If spin-orbit coupling is strong enough $\left(\alpha_{s o} \gg \alpha_{s p}\right), \theta_{c}$ becomes $\sim \frac{\pi}{2}$, i.e., the state approaches a unitary state $\Phi_{\|}(\mathbf{k}) \hat{\mathbf{z}}$. Whereas, if spin-orbit coupling is sufficiently weak $\left(\alpha_{s o} \leq \alpha_{s p}\right), \theta_{c}$ deviates from $\frac{\pi}{2}$ and then a non-unitary state $\Phi_{\|}(\mathbf{k})(\hat{\mathbf{z}}+i \alpha \hat{\mathbf{y}})$ is formed. Below $t_{c}$, free energy is obtained by minimizing eq. (7) with respect to $\theta$. From eq. (7), one can see that $\theta$ approaches $\frac{\pi}{2}$ much below $t_{c}$, and the unitary state $\Phi_{\|}(\mathbf{k}) \hat{\mathbf{z}}$ is formed irrespective of the value of $\alpha_{s p}$.

From the minimized free energy, we obtain the temperature dependence of the specific heat $C$ for various fields, which is shown in Fig. 2. Here, in order to obtain $C$ correctly to the order of $t_{c}-t$, we have perturbatively included the sixth order term $\frac{31 \zeta(5)\left\langle\Phi_{\|}(\mathbf{k})^{6}\right\rangle}{32 \pi^{4} T_{c 0}^{4}}|\bar{\Psi}|{ }^{6} r^{6}\left(1+3 \sin ^{2} 2 \theta\right)$ in the free energy. As shown in Fig. 2, $C$ has two peaks at high magnetic fields, while only a single peak at low fields, consistent with the experiments. In order to characterize the crossover region, we define a crossover temperature $t^{*}$ and a crossover magnetic field $H^{*}$ where $C$ takes the minimum. $t^{*}$ s are shown with arrows in Fig. $2 . t^{*}\left(H^{*}\right)$ can be associated with the starting point of the crossover with decreasing temperature (field). In Fig. 3, we plot $H^{*}(T)$ in $T$ $H$ diagram. $H^{*}(T)$ line is located just below $H_{c 2}$, and is terminated at $T \sim T_{t}=0.50 T_{c 0}$. Since the double peak structure becomes unobservable below $T_{t}$, this point can be naturally identified with the observed "tricritical point" as shown by an arrow in Fig. 3. This point corresponds to $\left(T_{t}, H_{t}^{*}\right)=(1.2 \mathrm{~K}, 0.8 \mathrm{~T})$. 
As to the field-angle dependence of the crossover behavior, we could not find any marked differences between $\phi_{0}=0$ and $\phi_{0}=\frac{\pi}{4}$. Actually, while the ac-susceptibility shows the characteristic field-angle dependence, ${ }^{8,9}$ the double-peak structure of specific heat does not change with field direction, thus consistent with our analysis.

Let us make some additional remarks. Firstly, the crossover discussed in this paper has a lot of features in common with the $\mathrm{A}_{1}-\mathrm{A}$ phase transition in ${ }^{3} \mathrm{He}$ under magnetic field. ${ }^{15-17}$ Due to a lack of spin-orbit coupling, a phase transition occurs in ${ }^{3} \mathrm{He}$ instead of a crossover. In order to discuss the $\mathrm{A}_{1}-\mathrm{A}$ transition of ${ }^{3} \mathrm{He}$ in our context, one only has to put $\alpha_{s o}=0$ in eq. (2). Then, one can find that the $\mathrm{A}_{1}-\mathrm{A}$ transition takes place at $t=t_{c}-2 \alpha_{s p}$. Secondly, our result does not deny the Agterberg's transition ${ }^{7}$ at low magnetic fields. Since the $k_{y}(\hat{\mathbf{z}}-i \alpha \hat{\mathbf{y}})$ state cannot be adiabatically continued to $\left(k_{x} \pm i k_{y}\right) \hat{\mathbf{z}}$ state, there still has to be a secondorder phase transition at a finite magnetic field up to $\mathrm{T}=\mathrm{T}_{c 0}$. Actually, it was found that magnetization shows a kink at low magnetic field, suggestive of the phase transition. ${ }^{18}$

Thirdly, we would like to comment on the zero-field state. So far, we have assumed that the $\left(k_{x} \pm i k_{y}\right) \hat{\mathbf{z}}$ state appears at zero magnetic field. However, this assumption is not necessary. For example, if the $k_{x} \hat{\mathbf{x}} \pm k_{y} \hat{\mathbf{y}}$ is stable and is nearly degenerate with $\left(k_{x} \pm i k_{y}\right) \hat{\mathbf{z}}$, one will find a crossover from $k_{y}(\hat{\mathbf{y}}+i \alpha \hat{\mathbf{z}})$ to $k_{y} \hat{\mathbf{y}}$, and double peaks appear in specific heat in the same way. Finally, the second-peak line of specific heat is placed at $H \sim 0.95 H_{c 2}$ at $T \sim 0$, which is lower than our theoretical estimate $H^{*} \sim 0.99 H_{c 2}$. However, in our analysis, we have not considered the renormalization of the effective mass $\left(m^{*}\right)$ and g-factor $\left(g^{*}\right)$. Due to the electron correlation, these factors are enhanced compared with the bare value as $\frac{m^{*}}{m_{\text {band }}} \sim 5.5$ and $\frac{g^{*}}{g_{\text {bare }}} \sim 1.5$, respectively. Since $\alpha_{s p}$ is proportional to the product $m^{*} g^{*}$, the renormalization of these values will push the crossover line down to lower field. Hence, the observed secondpeak line can be reproduced even quantitatively.

When a magnetic field is applied slightly off the plane, it is observed that the double peaks of the specific heat disappear. ${ }^{9}$ Currently, our theory cannot explain this phenomenon. However, the deviation of $\mathrm{H}_{c 2}$ line from the result of Ginzburg-Landau anisotropic effective mass model implies that we need to take account of the layered structure of this material in order to reproduce this phenomenon. We would like to leave this problem for future task.

In summary, we have shown that the observed double peaks of specific heat in $\mathrm{Sr}_{2} \mathrm{RuO}_{4}$ can be attributed to the crossover from $k_{y}(\hat{\mathbf{z}}-i \alpha \hat{\mathbf{y}})$ to $k_{y} \hat{\mathbf{z}}$ state. Within our theory, we could also explain the disappearance of double peaks at low magnetic fields.

We would like to thank M. Ichioka, M. Arai, and S. Yoshio for valuable discussions. 


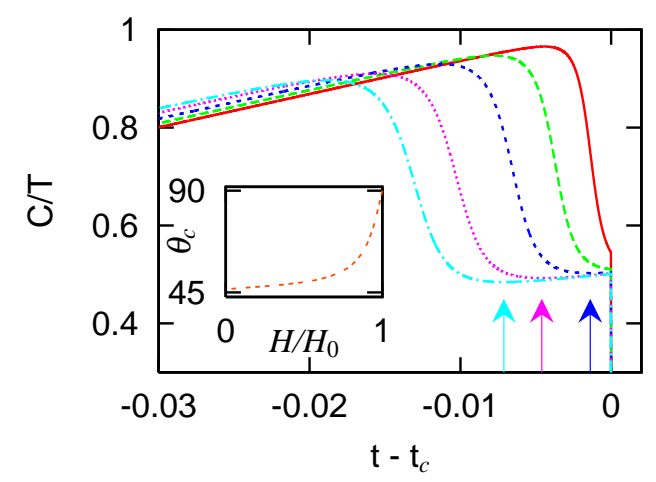

Fig. 2. Temperature dependence of the specific heat for $\frac{H}{H_{0}}=0.20,0.40,0.60,0.80,0.90$ from right to left. $\frac{C}{T}$ is normalized so that the jump at $t=t_{c}$ is 1 when $\alpha_{s p}=0$. The crossover temperature, $t^{*}$, are shown with arrows for $\frac{H}{H_{0}}=0.60,0.80$, and 0.90 . For other fields, $t^{*}$ is absent. The inset shows the $H$ dependence of $\theta_{c}$.

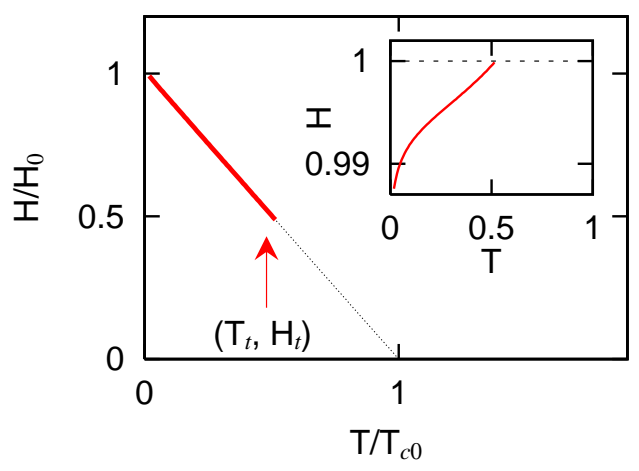

Fig. 3. $\mathrm{H}^{*}(T)$ line is shown with a red line in the $T-H$ diagram. A black line shows $H_{c 2}$ line. The "tricritical point", $\left(T_{t}, H_{t}\right)$, is shown with an arrow. (Inset) Same with the main panel, with $H$ normalized with $H_{c 2}$ of each temperature. 


\section{References}

1) Y. Maeno, H. Hashimoto, K. Yoshida, S. NishiZaki, T. Fujita, J. G. Bednorz and F. Lichtenberg: Nature 372 (1994) 532.

2) A. P. Mackenzie and Y. Maeno: Rev. Mod. Phys. 75 (2003) 657.

3) G. M. Luke, Y. Fudamoto, K. M. Kojima, M. I. Larkin, J. Merrin, B. Nachumi, Y. J. Uemura, Y. Maeno, Z. Q. Mao, Y. Mori, H. Nakamura and M. Sigrist: Nature 394 (1998) 558.

4) T. Nomura and K. Yamada: J. Phys. Soc. Jpn. 71 (2002) 1993.

5) Y. Yanase and M. Ogata: J. Phys. Soc. Jpn. 72 (2003) 673.

6) K. Deguchi, Z. Q. Mao, H. Yaguchi, and Y. Maeno: Phys. Rev. Lett. 92 (2004) 047002.

7) D. F. Agterberg: Phys. Rev. Lett. 80 (1998) 5184.

8) S. NishiZaki, Y. Maeno and Z. Q. Mao: J. Phys. Soc. Jpn. 69 (2000) 572.

9) K. Deguchi, M. A. Tanatar, Z. Q. Mao, T. Ishiguro and Y. Maeno: J. Phys. Soc. Jpn. 71 (2002) 2839.

10) M. A. Tanatar, S. Nagai, Z. Q. Mao, Y. Maeno and T. Ishiguro: Phys. Rev. B 63 (2001) 064505

11) Z. Q. Mao, Y. Maeno and H. Fukazawa: Mater. Res. Bull. 35 (2000) 1813

12) R. P. Kaur, D. F. Agterberg and H. Kusunose: cond-mat/0503289

13) H. Murakawa, K. Ishida, K. Kitagawa, Z. Q. Mao and Y. Maeno: Phys. Rev. Lett. 93 (2004) 167004

14) Since the Fermi surface is not round, the pairing function with the shortest coherence length is not $\Psi_{\|}(\mathbf{k})$. However, we ignore the error arising from this.

15) D. D. Osheroff, W. J. Gully, R. C. Richardson and D. M. Lee: Phys. Rev. Lett. 29 (1972) 920

16) V. Ambegaokar and N. D. Mermin: Phys. Rev. Lett. 30 (1973) 81

17) W. F. Brinkman and P. W. Anderson: Phys. Rev. A 8 (1973) 2732

18) K. Tenya, S. Yasuda, M. Yokoyama, H. Amitsuka, K. Deguchi and Y. Maeno: to appear in Physica B. 